\title{
VIABILIDADE DO CAPTOPRIL SUBLINGUAL EM CRISES HIPERTENSIVAS
}

VIABILITY OF SUBLINGUAL CAPTOPRIL IN HYPERTENSIVE CRISES

\author{
DIAS, Karina Gualqui Marchi ${ }^{1}$; DIETRICH, Karyne Ansante ${ }^{1}$; PEDRO, Marcela \\ Corrêa $^{1}$; MENESES, Eli Cristiano ${ }^{2}$ \\ ${ }^{1}$ Graduadas do Curso de Farmácia - Universidade São Francisco; \\ ${ }^{2}$ Docente do Curso de Farmácia - Universidade São Francisco
}

\section{k.gualqui@hotmail.com}

RESUMO. A Hipertensão Arterial é uma doença crônica, com origem por vários fatores, hereditários, idade, raça, hábitos alimentares e mobilidades físicas, provocando anomalias nos vasos sanguíneos, provocando uma alteração dos níveis de pressão arterial e muitas vezes provoca crises abruptas das quais retiram o paciente da homeostasia. As alterações dos níveis de pressão, obstruções arteriais, contribuem ao aparecimento de crises hipertensivas, infarto agudo do miocárdio, insuficiência cardíaca congestiva e AVC. Uma das classes indicadas para tratar esta patologia é a classe medicamentosa dos Inibidores da Enzima de Conversão da Angiotensina (IECA's) I e II. O medicamento desta classe mais utilizado é o captopril. Entretanto apesar do medicamento possuir uma forma farmacêutica desenvolvida e fabricada para administração via oral, ele vem sido utilizado a muitos anos pela via sublingual em emergências hipertensivas. Este segmento dá-se devido a sua eficácia significativa através desta via de administração. Após inúmeros testes realizados, pesquisadores acreditam que o medicamento tem efeito superior em relação ao tempo de eficácia, quando comparado a administração do mesmo por via oral.

Palavras-chave: Captopril; Sublingual; Crises hipertensivas; Farmacologia; IECA's.

ABSTRACT. The Arterial Hypertension is a chronic disease that often causes abrupt crises withdrawing the patient from homeostasis. One of the classes indicated to treat this pathology is a drug class of ACE inhibitors, angiotensin I and II inhibitors. The most commonly used medicinal product of this class is captopril. However, although the drug has a pharmaceutical form developed and manufactured for oral administration, it has been used for many years by the sublingual route in hypertensive emergencies. This segment is due to its significant efficacy through this route of administration. After numerous tests, researchers believe that the drug has a superior effect in relation to the time of effectiveness, when compared to oral administration.

Keywords: Captopril; Sublingual; Hypertensive crises; Pharmacologia; IECA's.

\section{INTRODUÇÃO}

A hipertensão arterial é uma patologia crônica que caracteriza-se pela elevação da pressão arterial. Esta pressão é a pressão que há no interior das artérias e faz comunicação com as paredes das mesmas (LOLIO, 1990). 
As crises hipertensivas ocorrem de maneira que a pressão arterial desencadeie uma elevação abrupta e pode causar diversos danos ao indivíduo. É considerado como número elevado da pressão arterial valores superiores a 140 mmHg (FRANCO, 2002).

Segundo a Organização Pan-Americana de saúde, a prevalência de hipertensão varia entre $22 \%$ e $44 \%$ em adultos (32\% em média) chegando a mais de $50 \%$ em indivíduos com 60 a 69 anos e $75 \%$ naqueles com mais de 70 anos (OPAS, 2016). Os casos de alterações da pressão arterial são mais prevalentes em: mulheres que fazem uso de medicamentos contraceptivos, fumantes, negros, pessoas de classe social mais baixas e indivíduos submetidos a um alto nível de estresse (ZAMPAGLIONE et al., 1996).

A abordagem ao paciente com crise hipertensiva deve abranger a história clínica, avaliação visual, pressórica, cardíaca (frequência cardíaca) e laboratorial, assim como também uma investigação de doença renal e história de cardiopatia. É importante salientar que existem duas questões a serem respondidas nos casos de crises hipertensivas, são elas: qual a velocidade da redução da pressão arterial e a proporção de sua redução. As respostas devem ser baseadas na teoria de que a redução dos níveis pressóricos deve ser lenta e contínua, até que a homeostasia seja obtida e que a segurança dos órgãos do paciente tenha sido colocada em evidência (OIGMAN, 2003).

Para que a hipertensão arterial seja restabelecida foi desenvolvido medicamentos denominados anti-hipertensivos. Dentre eles é possível encontrar o Captopril, medicamento pertencente à classe dos Inibidores de Enzima Conversora da Angiotensina (IECA). O mecanismo de ação dessas substâncias é fundamental dependente da inibição da enzina conversora (ECA), bloqueando a transformação de angiotensina I e II, no sangue e nos tecidos, proporcionando a diminuição dos níveis de angiotensina II e aldosterona e a pressão arterial é reduzida por diminuição de resistência vascular periférica, reduzindo a pressão arterial (BRUNTON, KNOLLMANN, 2018).

Os IECA's foram desenvolvidos por Ferreira, através do extrato do veneno da jararaca-da-mata (Botrops jararaca), no ano de 1960. Ferreira pôde perceber que este veneno intensificava a resposta vasodilatadora à enzima bradicinina, destruindo-a. Com isso ele pôde ajudar diversas pessoas com hipertensão arterial essencial (BRUNTON, KNOLLMANN, 2018).

Os inibidores da ECA são vasoconstritores potentes, pois impedem a conversão de angiotensina I em angiotensina II, produzindo assim seus efeitos. O uso desse inibidor é a melhor alternativa de medicação por via oral ou sublingual para o tratamento de crises hipertensivas, em que o paciente apresenta insuficiência cardíaca congestiva, AVC, hipertensão arterial maligna e infarto agudo do miocárdio (MARTELLI et al., 2008).

O comprimido de Captopril quando administrado por via oral, leva em torno de 1 (uma) a 2 (duas) horas para que após sua metabolização no organismo, apresente ação desejada. Isso resultaria em uma alternativa desfavorável diante de emergências hipertensivas, onde espera-se que o resultado do valor da pressão arterial se restabeleça o mais rápido possível, para que o paciente volte a homeostasia (KARLBERG et al., 1980).

Entretanto, quando testado em administração sublingual o medicamento devido ao contato direto com a mucosa da cavidade bucal atinge diretamente a corrente sanguínea, transportando-o através das veias e em seguida drenando-o para circulação sistêmica (NARANG; SHARMA, 2011), fazendo com que o espaço de tempo da administração até o início do resultado desejado seja de 5 (cinco) minutos. Essa 
constatação resultou em um grande salto para os tratamentos nas emergências referentes a elevação da pressão arterial (TSCHOLLAR; BELZ, 1985).

Apesar do uso do Captopril sublingual ter suas vantagens potencializadas pelo contato com a mucosa bucal, existem algumas desvantagens quanto a utilização de medicamentos através da via sublingual, dentre elas destacam-se a impossibilidade do uso em pacientes inconscientes e não cooperativos; assim como também os indivíduos não devem fumar ou ter fumado em um período próximo à administração do medicamento, pois o tabaco causa vasoconstrição resultando na diminuição da absorção do medicamento (NARANG; SHARMA, 2011).

A elevação da pressão arterial é grave e pode lesar os órgãos do indivíduo, levando-o até mesmo a óbito. Sendo assim é necessário que o tratamento tenha efeito em minutos ou no máximo em até uma hora após o início da crise hipertensiva (CHETTY et al., 2001). A descoberta da eficácia e rapidez do tratamento para Hipertensão Arterial Sistêmica (HAS) utilizando o Captopril pela via sublingual, deu-se porque foi possível verificar que a concentração plasmática do medicamento no organismo atingiu seu nível terapêutico em tempo inferior a via convencional, que teoricamente seria a via recomendada para a formulação na qual o medicamento é fabricado, porque o princípio ativo não realiza metabolização de primeira passagem para depois demonstrar seus efeitos terapêuticos no organismo (AL-FURAIH et al., 1991).

Foram realizados diversos estudos para verificar a veracidade da diferença dos efeitos farmacológicos do captopril em relação ao tempo e concentração plasmática quando administrado através de vias distintas (neste caso por via sublingual e via oral). Neste estudo foi possível observar que a concentração do captopril administrado por via sublingual atingiu valores superiores em tempo inferior quando comparado aos valores obtidos pelo captopril administrado por via oral (AI-FURAIH et al., 1991).

A utilização dos inibidores de angiotensina I e II interferem diretamente no comportamento dos níveis de renina circulantes no plasma (RODRIGUES; ALMEIDA, 2002). Através desta constatação é possível pautar que segundo eles quando o aumento da concentração de Captopril no plasma eleva-se, os níveis de renina presentes no plasma acompanham o aumento e a queda da concentração do medicamento no sistema endócrino do paciente (RODRIGUES; ALMEIDA, 2002).

Os exames de pacientes que apresentam análise preliminar de risco (APR) elevada mostram que estes são os indivíduos com maior probabilidade de manifestar complicações no sistema cardiovascular, incluindo infarto agudo do miocárdio, ressaltando a importância da rápida recuperação dos níveis pressóricos (BLUMENFELD et al., 2000).

Apesar da comprovação de que o captopril sublingual tem efeitos superiores em relação ao tempo de diminuição da pressão arterial, alguns pesquisadores ainda sugerem que os estudos realizados não foram suficientes para esta afirmação (DESSI-FULGHERI et al., 1987). Embora esta via seja muita das vezes a via de escolha de muitos médicos, ela acaba sendo inviável pelo sabor desagradável e por causar em vários casos hipersensibilidade local e queimaduras químicas na mucosa oral (KARAKILIÇ et al., 2012), e outros estudos mostram que o uso de drogas cardiovasculares acarretou perda de sabor e paladar aos pacientes submetidos a esses processos de tratamento (ZERVAKIS et al., 2000). 
Pesquisadores sugerem que sejam feitas análises de lesão de órgão-alvo progressivas para verificar a necessidade de uma diminuição abrupta da pressão arterial do paciente. Caso a resposta seja negativa para essas lesões, os estudos recomendam que esta diminuição seja feita de forma lenta e contínua, descartando a necessidade da utilização do captopril sublingual (KARAKILIÇ et al., 2012).

O objetivo do presente trabalho é avaliar a viabilidade do uso do comprimido de captopril por via sublingual em crises hipertensivas nas unidades emergenciais.

\section{MATERIAIS E MÉTODOS}

Para a realização da pesquisa do tema descrito neste trabalho, utilizou-se o método de revisão bibliográfica, visando entender e explorar o assunto de forma que fosse possível analisar e debater o surgimento da prática adotada em relação a administração do captopril em emergências hipertensivas.

Toda a pesquisa teve embasamento científico e foi realizada com base em artigos e fontes secundárias, como trabalhos acadêmicos a respeito do tema. Este embasamento científico foi realizado através dos bancos de dados da Scielo e do Google Acadêmico, onde foram encontrados recursos suficientes e necessários para a elaboração do projeto, pois os mesmos possuem um amplo campo de artigos repletos de conhecimento e pesquisas inseridos em seus sistemas.

Todos os artigos e trabalhos secundários escolhidos, enquadram-se perfeitamente no tema e foram citados em diversos artigos vinculados a esta proposta, recuperando conhecimento científico acumulado sobre o tema do projeto.

\section{RESULTADOS E DISCUSSÃO}

Apesar da hipertensão arterial caracterizar-se como uma doença sistêmica grave, quando se instauram medidas de contenção para reverter tais crises hipertensivas, é possível fazer com que seja possível estabelecer níveis pressóricos estáveis e aceitáveis através de um tratamento adequado para cada indivíduo, de acordo com suas necessidades.

Tendo em vista os estudos e artigos utilizados para esta revisão bibliográfica, é possível salientar que a utilização do Captopril tem efeito significativo quando direcionado à aplicação pela via sublingual. Tal afirmação pode ser atestada através da análise dos estudos realizados por AI-Furaih e seus colegas, que comprovam através do estudo científico que resultou no artigo "Sublingual captopril- a pharmacokinetic and pharmacodynamic evaluation" de 1990, a efetividade do captopril sublingual, assim como também no artigo "Sublingual captopril in hypertensive crisis" de 1985 realizado por Tschollar e Belz.

A biotransformação do Captopril sublingual não é um aspecto que varia quando aplicada em diferentes vias de utilização, pois o mesmo não necessita de biotransformação para desempenhar sua função e isso gera maior segurança em relação a esta aplicação sublingual.

A forma farmacêutica do medicamento Captopril não é elaborada para a aplicação em via sublingual, entretanto alguns estudos comprovam que este medicamento apesar da utilização realizada em uma via onde a forma farmacêutica não é a recomendada, traz 
efeitos benéficos ao paciente. Segundo os artigos estudados, em casos de crises hipertensivas emergenciais é possível dizer que esta prática é segura.

É indispensável ressaltar que os exames laboratoriais que detectam os níveis de atividade de renina plasmática são de extrema importância para a avaliação de risco do paciente, pois o Captopril sublingual é refém do efeito rebote por baixar a pressão arterial em menor espaço de tempo e por ter a concentração plasmática de forma mais rápida, sendo assim outras medidas de contenção da pressão arterial devem ser aplicadas.

Apesar do Captopril sublingual ser muito utilizado para obtenção de resultados rápidos, seus efeitos podem não ser apenas benéficos, pois o mesmo pode apresentar gosto amargo e causar efeitos indesejáveis como hipersensibilidade local e hipogeusia. Alguns estudos sugeriram que o medicamento aplicado na via sublingual pode causar males irreversíveis aos pacientes que fazem o uso contínuo por esta via. Segundo os pesquisadores Zervakis, Graham e Schiffman autores do artigo "Taste effects of lingual application of cardiovascular medications" do ano de 2000, com base em seus estudos foi possível verificar que incialmente o Captopril sublingual agride a detecção de sabores doces e salgados em nossas papilas gustativas. A longo prazo todos os quatro sentidos que temos (amargo, doce, salgado e azedo) também é afetado.

Esta questão faz com que deva ser feita uma investigação inicial (antes da aplicação do medicamento) a respeito de lesões de órgão-alvo progressiva (se existentes), e em caso de ausência de lesões, o restabelecimento da pressão arterial do paciente deve ser feito de modo lento e progressivo, gerando menos efeitos adversos ao mesmo, assim como a orientação ao paciente de que o mesmo deve manter usualmente a utilização do Captopril por via oral, deixando claro que a utilização por via sublingual deve ser realizada somente em casos de urgências e emergências hipertensivas e sob supervisão de uma equipe médica.

\section{CONCLUSÃO}

A hipertensão arterial é uma doença que acomete milhares de pessoas no mundo todo, seja ela pelos mais diversos motivos, comorbidades, hereditariedade, idade, stress, emoções como perda de familiares ou por hábitos alimentares e falta de atividades físicas e emergências hipertensivas ocorrem diariamente em setores de atendimento à saúde, sejam eles pronto atendimento, hospitais ou clínicas.

Os órgãos públicos e complementares estão muito atentos a esse mal que acomete a população, em todos centros de saúde e ambulatórios de doenças cardiovasculares, quer público (SUS) ou saúde complementar são realizados palestras, oficinas e muitas outras atividades para conscientização desses pacientes, com objetivo de ter uma qualidade de vida melhor e não sobrecarreguem o sistema de saúde.

O Captopril é muito utilizado nas emergências hipertensivas, e sua utilização por via sublingual é a opção, quando nível de pressão pode ser tratados em ambulatórios. Com base nos estudos que foram realizados é possível comprovar a eficácia do medicamento por essa via, entretanto é necessário que sejam realizados outros estudos que abranjam a magnitude dos efeitos colaterais aos pacientes que fazem uso do Captopril por via sublingual.

Portanto espera-se entender o risco benefício do medicamento e o porquê da não produção do mesmo em comprimidos sublinguais. 


\section{REFERÊNCIAS}

AL-FURAIH, T. A. et al. Sublingual captopril-a pharmacokinetic and pharmacodynamic evaluation. European journal of clinical pharmacology, v. 40, n. 4, p. 393-398, 1991.

BLUMENFELD, J. D. et al. Plasma renin activity in the emergency department and its independent association with acute myocardial infarction. American journal of hypertension, v. 13, n. 8, p. 855-863, 2000.

BRUNTON, Laurence L.; KNOLLMANN, Björn C. As Bases Farmacológicas da Terapêutica de Goodman e Gilman-13. Artmed Editora, 2018.

CHETTY, D. J.; CHEN, L. H.; CHIEN, Y. W. Characterization of captopril sublingual permeation: determination of preferred routes and mechanisms. Journal of pharmaceutical sciences, v. 90, n. 11, p. 1868-1877, 2001.

DESSI-FULGHERI, P. et al. Comparison of sublingual and oral captopril in hypertension. Clinical and Experimental Hypertension. Part A: Theory and Practice, v. 9, n. 2-3, p. 593-597, 1987.

FRANCO, R. J. S. Crise hipertensiva definição, epidemiologia e abordagem diagnóstica. Rev Bras Hipertens, v. 9, n. 4, p. 340-5, 2002.

KARAKILIÇ, E. et al. Same effect of sublingual and oral captopril in hypertensive crisis. Eur Rev Med Pharmacol Sci, v. 16, n. 12, p. 1642-5, 2012.

KARLBERG, B. E. et al. Captopril lowers urinary kallikrein in hypertensive patients. The Lancet, v. 315, n. 8160, p. 150-151, 1980.

MARTELLI, Anderson; LONGO, Marco Aurélio Tosta; SERIANI, Cleber. Aspectos clínicos e mecanismo de ação das principais classes farmacológicas usadas no tratamento da hipertensão arterial sistêmica. Estudos de Biologia, v. 30, n. 70/72, 2008.

NARANG, N.; SHARMA, J. Sublingual mucosa as a route for systemic drug delivery. Int J Pharm Pharm Sci, v. 3, n. Suppl 2, p. 18-22, 2011.

OIGMAN, W. Crise hipertensiva: quando e como abordá-la. Revista da SOCERJ, v. 16, n. 1, p. 52-9, 2003.

RODRIGUES, C. I. S.; ALMEIDA, F. A. Valor e limitações das dosagens de renina plasmática na prática clínica. Rev Bras Hipertens, v. 9, n. 2, p. 203-5, 2002. 


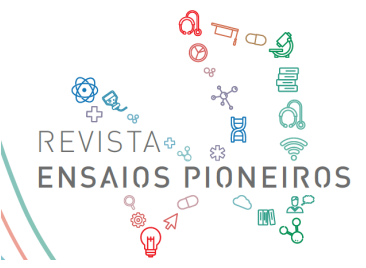

http://ensaiospioneiros.usf.edu.br

TSCHOLLAR, W.; BELZ, G. G. Sublingual captopril in hypertensive crisis. The Lancet, v. 326, n. 8445, p. 34-35, 1985.

ZAMPAGLIONE, B.; PASCALE, C.; MARCHISIO, M.; CAVALLO-PERIN, P. Hypertensive urgencies and emergencies: prevalence and clinical. Hypertension 1996; 27(1):144-147.

ZERVAKIS, J.; GRAHAM, B. G.; SCHIFFMAN, S. S. Taste effects of lingual application of cardiovascular medications. Physiology \& behavior, v. 68, n. 3, p. $405-$ $413,2000$.

Publicado em 27/08/2020 